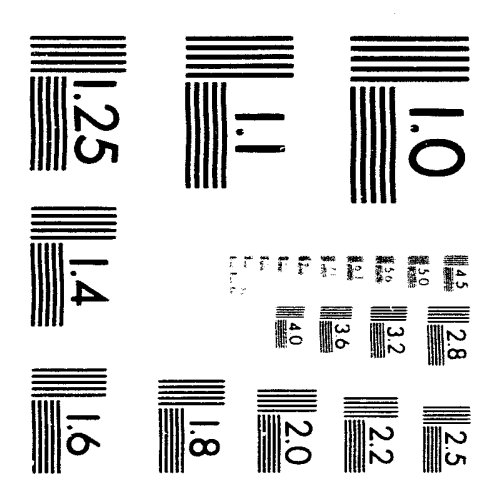



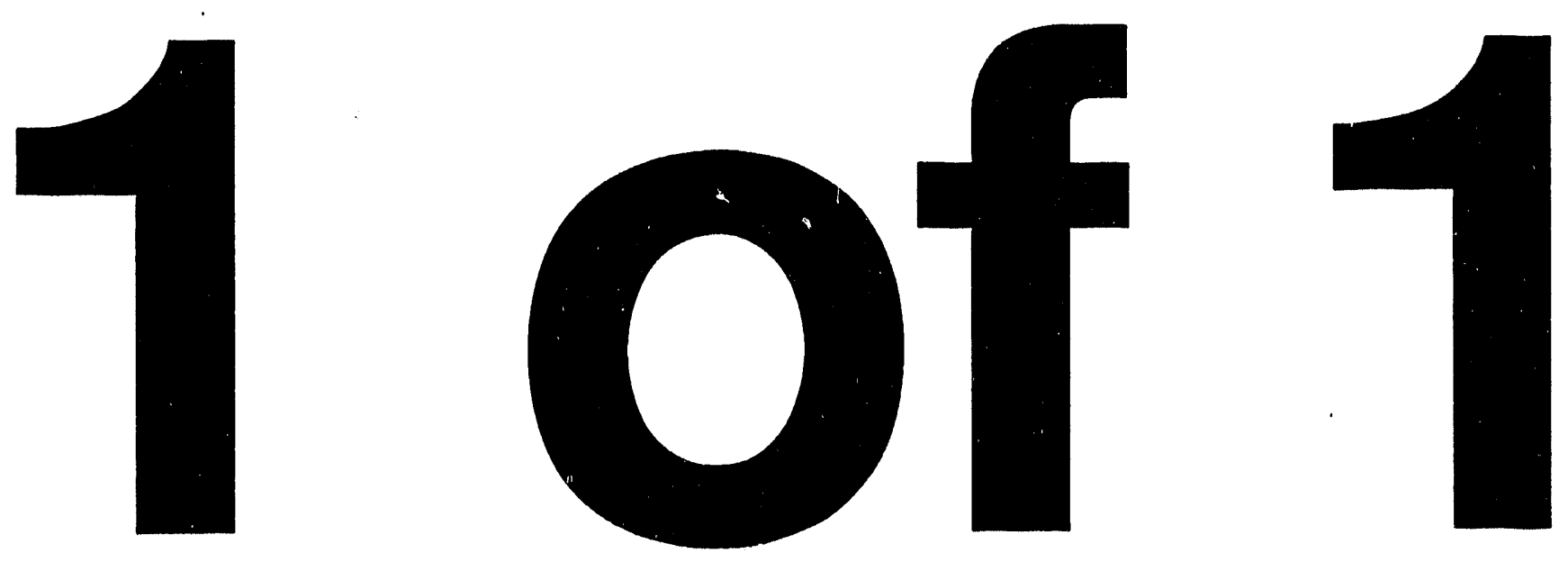


\title{
ADVANCES IN PASSIVE-REMOTE AND EXTRACTIVE FOURIER TRANSFORM INFRARED SPECTROSCOPIC SYSTEMS*
}

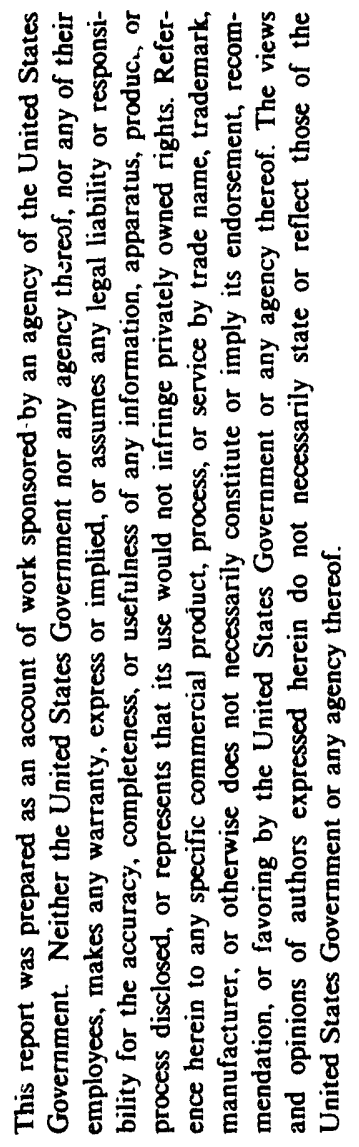

\author{
Jack C. Demirgian, Cheryl Hammer, and Elizabeth Hwang \\ Argonne National Laboratory \\ Analytical Chemistry Laboratory \\ Chemical Technology Division \\ 9700 South Cass Avenue \\ Argonne, IL 60439 \\ and \\ Zhuoxiong Mao \\ Argonne National Laboratory \\ Energy Systems Division \\ 9700 South Cass Avenue \\ Argonne, IL 60439
}

To be presented at

Air \& Waste Management Association's

International Specialty Conference

October 11-14, 1993

Atlanta, GA

The submitted manuscript has been authored
by a contractor of the U.S. Government
under contract No. W-31-109-ENG.38.
Accordingly, the U. S. Government retains a
nonexclusive, rovalty-free license to publish
or reproduce the published form of this
contribution, or allow others to do so. for
U.S. Government purposes.

"Work supported by the U.S. Department of Energy under Contract W-31-109-Eng-38. 


\title{
Advances in Passive-Remote and Extractive Fourier Transform Infrared Spectroscopic Systems
}

\author{
Jack Demirgian, Cheryl Hammer, Elizabeth Hwang, Chemical Technology Division \\ Zhuoxiong Mao, Environmental Systems Division \\ Argonne National Laboratory \\ 9700 South Cass Avenue \\ Argonne, IL 60439
}

\begin{abstract}
The Clean Air Act of 1990 requires the monitoring of air toxics including those from incinerator emissions. Continuous emission monitors (CEM) would demonstrate the safety of incinerators and address public concern about emissions of hazardous organic compounds. Fourier transform infrared (FTIR) spectroscopy can provide the technology for continuous emission monitoring of stacks. Stack effluent can be extracted and analyzed in less than one minute with conventional FTIR spectrometers. Passive-remote FTIR spectrometers can detect certain emission gases over $1 \mathrm{~km}$ away from a stack.

We will discuss advances in both extractive and passive-remote FTIR technology. Extractive systems are being tested with EPA protocols, which will soon replace periodic testing methods. Standard operating procedures for extractive systems are being developed and tested. Passive-remote FTIR spectrometers have the advantage of not requiring an extracted sample; however, they have less sensitivity. We have evaluated the ability of commercially available systems to detect fugitive plumes and to monitor carbon monoxide at a coal-fired power plant.
\end{abstract}

\section{INTRODUCTION}

Fourier transform infrared spectroscopy is a promising technology for identifying and quantifying incinerator emissions. This technology takes advantage of the high sensitivity and selectivity of infrared absorption bands in the "fingerprint" region $(8-12 \mu \mathrm{m})$, which is also in an atmospheric transmission window. In the last year hand-held FTIR spectrometers have become commercially available, and the development of better ways of obtaining the data quality control (QC) required by the Environmental Protection Agency (EPA) have been realized. These advances will make it possible to continuously monitor incinerator emissions in real time. Both extractive and passive-remote spectrometers are being developed for this purpose.

Currently, there is no on-stream method of monitoring incinerator exhaust emissions to determine whether hazardous substances such as benzene, methane, or ethylene have been destroyed or whether other hazardous products of incomplete combustion (PICs) are present. An on-stream CEM would demonstrate the safety and reliability of incinerators. The instrument would differentiate species in the parts-per-million (ppm) and parts-per-billion (ppb) range and calculate the destruction and removal efficiency. This information can be used to address reasonable public concern about incinerator safety and to smooth the process of obtaining permits.

A conventional extractive FTIR spectrometer contains an infrared source, an interferometer, focusing optics, a detector, and a heated long path-cell. The sample enters and exits the long path cell. Identification of the components in the sample is based upon a unique spectral "fingerprint." Quantitation is based upon Beer's law, which assumes that the amplitude of the spectral absorbance is linearly dependent on concentration. Overlapping spectral absorbances are resolved through the use of classical least square (CLS) and partial least square (PLS) algorithms. It is possible to obtain quantitative data because the sample and source temperatures are constant.

The same analytical tools developed for conventional FTIR spectroscopy can be applied to passive-remote FTIR spectroscopy, which makes use of mobile instruments that are capable of 
detecting organic and some inorganic plumes. Unlike conventional FTIR instruments, they do not contain an infrared source or a sample cell. These spectrometers are equipped with spectral input optics that collect ambient infrared light. Absorption or emission of infrared energy by the sample, relative to the infrared background, is observed by the instruments detector. It is not currently possible to calculate concentrations from remote data because the temperatures of the plume and the ambient energy source are not known and are not necessarily constant.

In addition to continuous monitoring, regulators need to independently determine whether all stack and fugitive emissions at a facility are in compliance with Title III requirements. Passiveremote FTIR technology offers the opportunity to identify and track plumes to their source and to independently monitor a source. The equipment has the additional advantage of being completely portable.

We will discuss tests in which we used transportable passive-remote units to monitor power plants and industrial sites for $\mathrm{CO}$ and $\mathrm{CO}_{2}$ emissions. We also tested the ability of the unit to detect plumes of released organics. The instrumentation detected a $10 \mathrm{~cm}, 19.4 \mathrm{ppm}-\mathrm{m}$ methanol plume from over $50 \mathrm{~m}$ away.

A new instrument is currently being tested that is extremely light and portable. It is slightly larger than a can of soda, weighs less than $1 \mathrm{lb}$, and will operate on a portable battery pack. This instrument should enable an individual to determine whether a facility is in compliance with the Clean Air Act easily and quickly.

\section{Experimental Setup}

The extractive FTIR system used in these experiments consisted of a Nicolet 8220 spectrometer (Nicolet Analytical Instruments, Madison, WI) interfaced to an Hanst 4-22 long-path cell (Infrared Analysis, Anaheim, CA) that was heated to $150^{\circ} \mathrm{C}$. The sample is brought to the longpath cell through a heated transfer line. Test results were obtained using a small laboratory incinerator in which the temperature and operating conditions can be altered reproducibly to simulate upset conditions. The CLS algorithm in the Nicolet PCIR software was used for quantitative data analysis. The apparatus and typical experimental conditions have been described previously (1).

Two passive-remote systems were tested. A commercially available Bomem spectrometer (Hartmann-Braun, Quebec) was tested outdoors for its plume detection capabilities. It was then used to detect $\mathrm{CO}$ at a power plant and in industrial areas. A miniFTIR spectrometer (Designs \& Prototypes [DP], Nashua, NH), which weighed less than $1 \mathrm{lb}$, was tested in the laboratory. The quantitative chemical releases for these tests were generated by a specially designed vaporizer which has been described previously (2). We used a notebook personal computer (PC) and SpectraCalc software (Galactic Industries, NH) to collect Bomem data. Data from the DP miniFTIR spectrometer were collected with proprietary vendor software and then converted to SpectraCalc format for further data analysis.

\section{Advances in Extractive Detection}

There has been much progress is the development of an FTIR CEM in the last year. The EPA directed Entropy Environmentalists, Inc., to develop a protocol for continuous monitoring that will eventually become the basis of performance specifications. The draft is available from the EPA upon request. We have applied this protocol to the data we obtained by using our laboratory incinerator and converted it into a step-wise standard operating procedure (SOP). This SOP is also available upon request. The protocol requires many statistical QC tests, which need to be programmed so they will run automatically. After this programming is done, much of the detailed analysis will be invisible to the user.

The new protocol has been tested at the Toxic Substances Control Act (TSCA) incinerator at the Oak Ridge Gaseous Diffusion Plant (K25) in Oak Ridge from August 23-September 2, 1993. The incinerator was burning mixed waste PCBs. The purpose of this test will be to demonstrate the ability of an FTIR CEM to operate over an extended period of time and to determine and focus upon 
the critical parameters. The results will be verified at the laboratory incinerator after the field test.

The FTIR data obtained at the TSCA incinerator at K25 will be used to identify and quantify: $\mathrm{HCl}, \mathrm{CO}$, benzene, toluene, methane, ethylene, and all one and two carbon chlorinated hydrocarbons. Currently detection levels are at the low ppm to high ppb levels. Due to some technological advances in data analysis that are under investigation these detection levels may be improved to the $10 \mathrm{ppb}$ level. Clean Air Engineering (Palatine, IL) provided the probe and transfer line.

If FTIR spectroscopy is to be accepted by EPA as a method for continuously monitoring emissions, the data must be analyzed at the same QC level as in other EPA-approved methods. Data quality is especially critical if FTIR spectroscopy is to be used in addressing the requirements of the Clean Air Act.

Conditions that affect data quality in FTIR spectroscopy include cell opcrating temperatures, corrosion of the cell, spectral interference, and spectral overlap. In an FTIR CEM, unlike an ambient-air FTIR system, the cell must be maintained at an elevated temperature, which increases system noise and makes cell alignment more difficult. Also, the cell is vulnerable to corrosion by acidic combustion products, especially $\mathrm{HCl}$, which is produced when PCBs are burned. Several cell components were tested for their ability to withstand heat and acidic conditions. The best results were obtained by using a glass-walled cell with gold-coated mirrors and anodized-aluminum hardware. When a teflon coating was used, $\mathrm{HCl}$ dissolved in the teflon and was subsequently outgassed. Stainless steel absorbed more gases than the anodized aluminum. Spectral interference and overlap are particularly important data quality issues. The effluent contains very high concentrations of water and $\mathrm{CO}_{2}$, which obscure relatively large spectral regions. Figure 1 shows a spectral background of incinerator effluent under highly efficient pure methane burn conditions. Only those regions with absorbance values less than 2 absorbance units (AU) can be used to analyze the products of combustion. In Figure 2, a spectrum from a "clean" burn (lower curve) can be compared to a spectrum taken when the incinerator is operating under upset conditions during a toluene burn (upper curve). After the incinerator was upset by lowering the operating temperature, PICs began to appear. The relative intensity of the PICs is low compared to the intensities of water and $\mathrm{CO}_{2}$, making them difficult to detect.

Figure 3 illustrates a further difficulty in identifying PICs: spectral overlap. The top spectrum is from a simulated PCB burn, the lower spectra are from analytical standards. By comparison, the PICs can be clearly identified in the burn spectrum; however, the complexity of the spectrum and the degree of overlap of the spectral absorbances requires fairly sophisticated data analysis.

Three methods of data analysis are available to overcome spectral overlap: CLS, PLS, and time-domain analysis. CLS requires a calibration curve for each pure component and works best when all components in the spectrum are in the spectral library. The software seeks a best fit by determining the combination of components having the smallest spectral residual. If the residual is high, the results are printed with a corresponding high error value. A more sophisticated data analysis method, such as PLS, requires calibration curves for both pure components and mixtures. The extra calibration requirements for PLS make it harder to use in the field. In systems in which component absorption peaks do not completely overlap, CLS and PLS predict virtually identical results. When there are multiple components that are not in the spectral library and there is severe spectral overlap, PLS outperforms CLS and reduces the error by half. It is much more difficult to develop a PLS spectral library because of the large number of mixtures required for accurate identification and quantitation.

A third method being developed is time-domain data analysis, which makes use of the interferogram instead of the infrared spectrum. This technique has tremendous potential for identifying selected target analytes in a complex mixture, because absorbances that overlap in the spectral domain may not overlap to the same degree in the time domain. Each point on the interferogram contains information about every spectral frequency, but in unequal amounts. Most of 
the broad spectral background information is represented at the centerburst. By digitally filtering the interferogram, the background can be drastically reduced, thereby eliminating the need to subtract the background or calculate a ratio of sample to background. Information about narrow spectral bands predominate further out in the wings of the interferogram, while information about broad spectral bands is closer to the centerburst. Hence, a narrow band that is completely covered by a broad band in the spectral domain can be separated in the time domain. For very broad bands, the spectral domain may be superior for data analysis, whereas the time domain may be most suitable for identifying narrow spectral bands.

The quantitative results from an FTIR CEM will probably be calculated by means of all three of these approaches. The analysis will be encoded on chips to facilitate automation. Earlier FTIR systems were removed from the market because of the complexity of the data analysis. The systems now' being tested are far more sophisticated and capable of much more complex computations.

\section{Advances in Passive-remote Detection}

Passive-remote FTIR spectrometers can identify Title III environmental air toxics in the presence of potentially interfering compounds. The technique is especially useful for the identification of a plume source and of the chemical components of a fugitive emission. In this section, we discuss a study in which we tested the capability of a commercially available, Bomem, passive-remote FTIR spectrometer to identify fugitive and stack emissions. Spectral data were obtained by subtracting the background spectrum from the sample spectrum. For releases produced by the laboratory vaporizer, the background spectrum was collected before initiating the releases. For stack emissions, we took the background by aiming upwind of the stack. Detection is most sensitive in the $8-12 \mu \mathrm{m}\left(1200-800 \mathrm{~cm}^{-1}\right)$ spectral region, where most organics absorb, and in the $2600-2000 \mathrm{~cm}^{-1}$ region, where $\mathrm{CO}_{2}$ and $\mathrm{CO}$ absorb.

Quantitation requires knowledge of the plume and background temperatures. The plume temperature can be determined from the hot bands of an analyte such as $\mathrm{CO}$. The background temperature can be obtained using a radiometer. Because both temperatures may be changing constantly, the calculation is more difficult and quite complex. Significant effort would be required to make it routine.

The concentration-path length $(C L)$ unit of ppm-m is used in stand-off detection because both the path length and the concentration are needed to represent the quantity of analyte present in the environment. A 1 ppm-m plume has a $C L$ of $1 \mathrm{ppm}$ and is $1 \mathrm{~m}$ thick. This plume contains the same number of molecules as a $10 \mathrm{ppm}$ plume that is $0.1 \mathrm{~m}$ thick. CO spectra obtained from stack ernissions are reported as qualitative data.

An initial data set was collected in the laboratory to provide a reference for outdoor emissions detection. Figure 4 shows spectra for three releases of methanol at 4.0, 9.25, and 19.4 ppm-m. A $51^{\circ} \mathrm{C}$ blackbody was used, and the plume temperature was $37^{\circ} \mathrm{C}$. The spectral resolution was $1 \mathrm{~cm}^{-1}$. The plumes were detected at a distance of $2 \mathrm{~m}$ from the release. The plume $w 1 \mathrm{~s} 10 \mathrm{~cm}$ high and $10 \mathrm{~cm}$ deep.

A 19.4 ppm-m methanol plume was released in front of a brick wall that was in the morning shade. The plume was monitored from $2 \mathrm{~m}$ away. Data were collected at a spectral resolution of 4 $\mathrm{cm}^{-1}$. The spectrum is shown in Figure 5. Because the background is now colder than the release, the methanol bands are in emission mode (pointing up). The instrument was later moved approximately $53 \mathrm{~m}$ from the release. Figure 6 shows the spectrum. By the time these data were collected, the brick wall was in the sun and hotter than the release. Hence, the spectrum is in adsorption mode (pointing down). The ability of commercial equipment to detect a $10-\mathrm{cm}$ plume at $53 \mathrm{~m}$ is impressive. The plume size is equivalent to a leak from a slightly opened window.

Figure 7 shows how peak intensity can vary with different backgrounds. Methanol was released immediately in front of the Bomem spectrometer, which was aimed at three backgrounds: grass, trees, and low sky. The difference in amplitude of the spectral bands for each of the backgrounds can be explained by the difference in temperature between each background. The 
greater the difference in temperature of the background and plume, the greater the amplitude of the absorbance band.

The Bomem spectrometer was also taken to a coal-fired power plant. The instrument was placed almost $1 \mathrm{~km}$ from the stack that was being monitored for $\mathrm{CO}$ emissions. The lower spectrum in Figure 8 represents the $\mathrm{CO}$ in the stack emissions. The $\mathrm{CO}$ can be clearly identified; however, fairly sophisticated data analysis was required because of the complexity of the spectrum and the degree of degradation of the spectral absorbances. Although the data were collected at $2 \mathrm{~cm}^{-1}$ resolution, the spectrum shows considerable spectral degradation. This degradation may have been caused by reabsorption by naturally occurring atmospheric $\mathrm{CO}$. It is also possible that the relatively slow stroke time of the interferometer may exceed the rate at which the radiant energy of the atmosphere changed.

Additional measurements were made at industrial sites in the Chicago area. Starks that were emitting $\mathrm{CO}$ were readily detectable. The spectra obtained were similar to Figure 8 . All data were collected at distances in excess of $1 \mathrm{~km}$ from the source. The technique was sensitive enough to track the source of the plume to the correct stack.

A prototype miniFTIR was also tested. The entire instrument weighs less than $1 \mathrm{lb}$, is slightly larger than a 12-oz can, and can be operated from a Poquet computer or laptop PC. The potential of the instrument lies in its portability. It can easily be carried to a site, while larger instruments would have to stay at a fence line. The DP spectrometer is less sensitive than the Bomem. Figure 9 shows a spectrum of a $19.4 \mathrm{ppm}-\mathrm{m}$ methanol plume collected in the lab. Further development of this instrument should greatly enhance its sensitivity. There is great potential for a hand-held FTIR spectrometer that can monitor emissions.

\section{CONCLLSION}

Extractive FTIR systems have advanced to the point where they are being tested for EPA acceptance. A protocol is now in place which will be the basis for EPA-certified performance specifications. Testing of the protocol will continue through this year. Difficulties with sampling and with identifying and quantifying complex spectral absorbances with overlapping bands are being overcome. In the next two years, data analysis should be at the same level as that required for other EPA-certified methods. During that time, the operating procedures must be simplified so monitoring can be performed by technicians.

Passive-remote FTIR systems are commercially available that can detect and identify plumes at $\mathrm{ppm}-\mathrm{m}$ concentrations from relatively far distance. The equipment cannot yet respond in real time, and it still requires spectral subtraction of the background. In the future, the instrumentation will be significantly reduced in size, and real-time data analysis will be possible. The technology being developed for the extractive FTIR CEM will be applied to passive-remote scenarios, which will allow data to be collected in real time. Additional analytical methods will be developed that will allow quantitation at the same QC level as other emissions-monitoring methods.

\section{REFERENCES}

1. Mao, Z., McIntosh, M., Demirgian, J., "On-line Emissions Monitoring of Chlorobenzene Incineration Using Fourier Transform Infrared Spectroscopy," Proceedings of the 1992 Incineration Conference, May 11-15, 1992, Albuquerque, NM, (1992).

2. Demirgian, J., Spurgash, S.; Remote Detection of Chemical Agents by Infrared Spectroscopy, ANL/ACL-90/1, Argonne National Laboratory, (February 1990). 


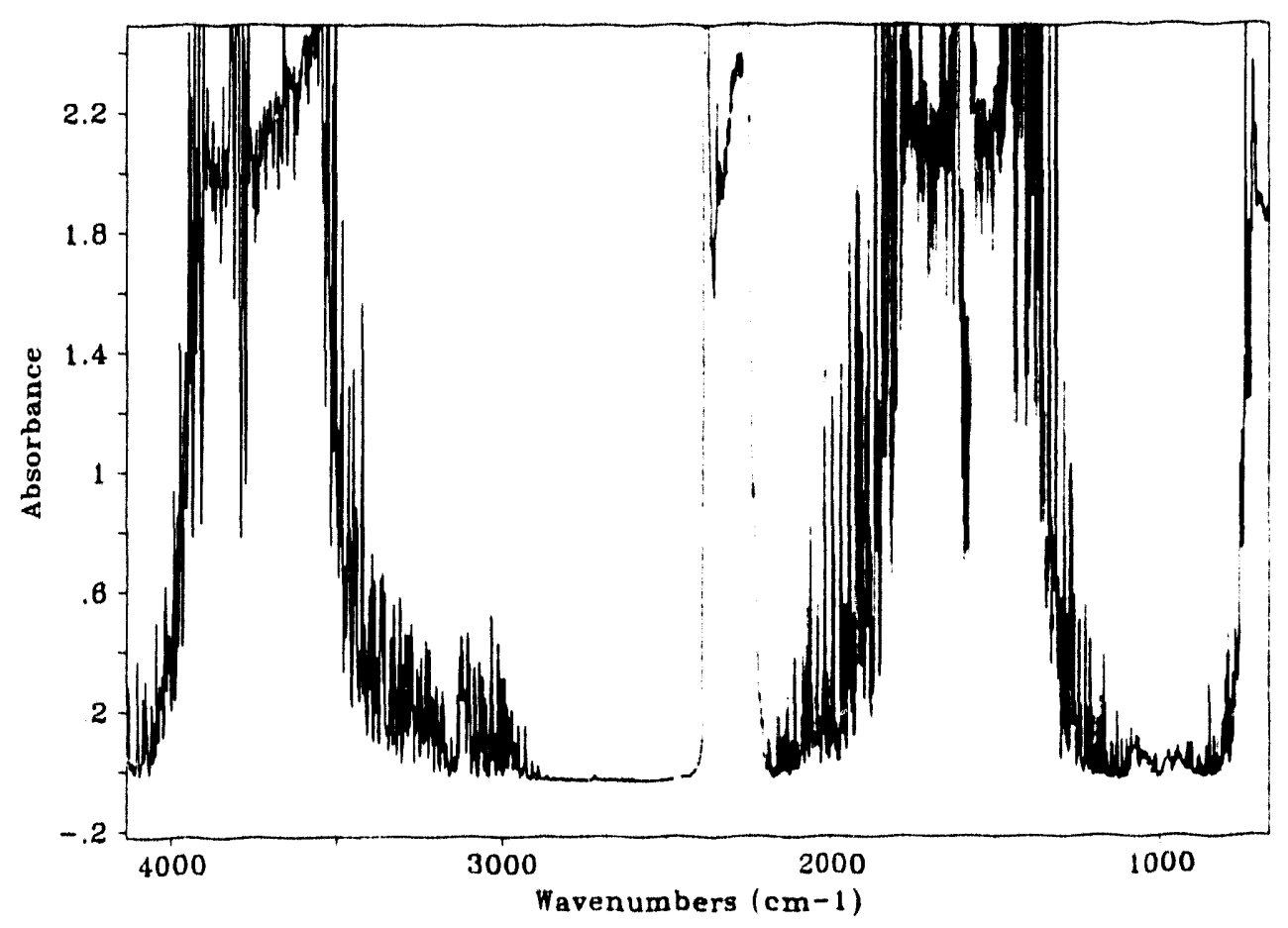

Figure 1. Infrared Spectrum of Incinerator Effluent with $99.9999 \%$ Bum Efficiency.

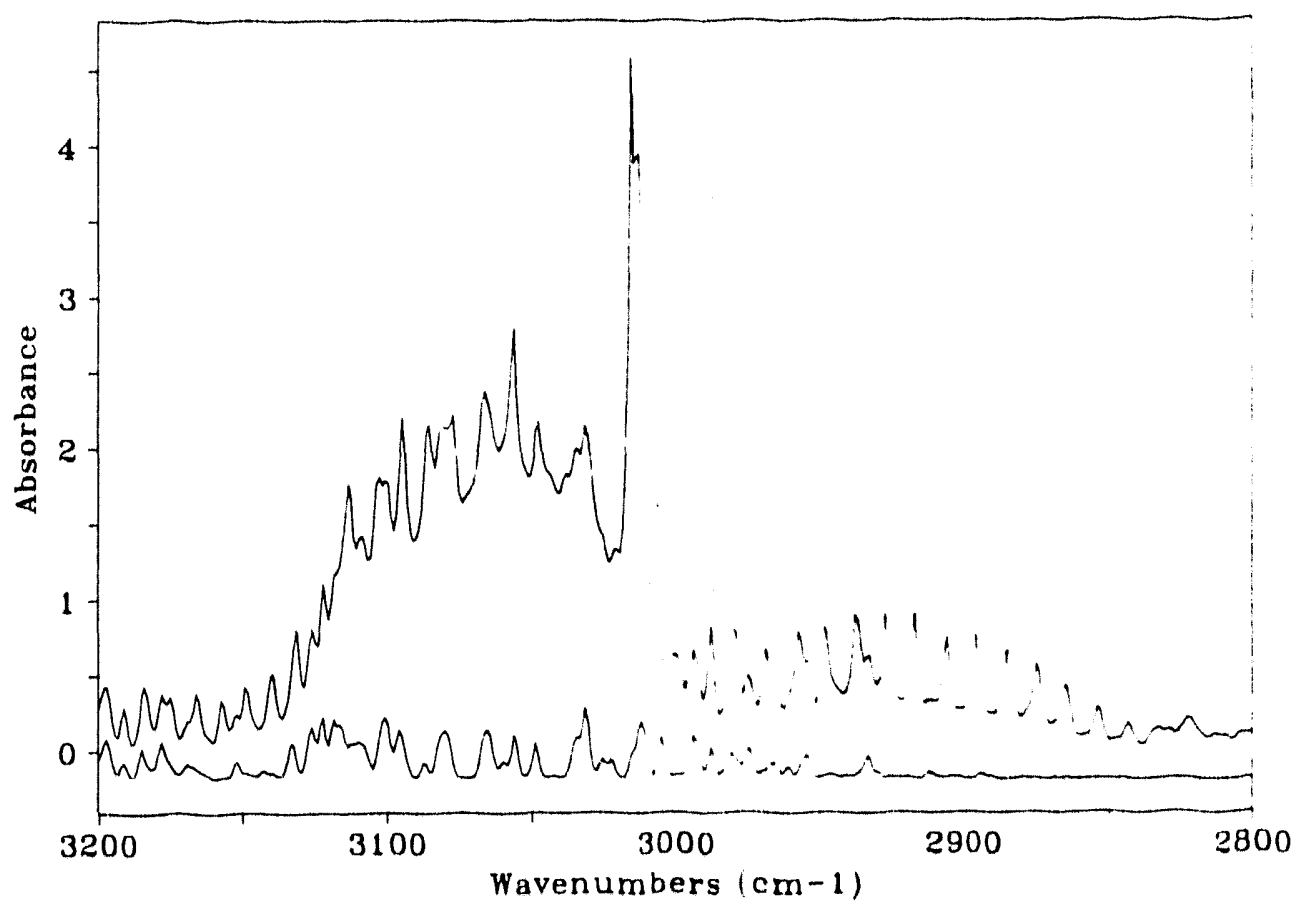

Figure 2. Comparison of Incinerator Emissions at $1100^{\circ} \mathrm{F}$ (lower curve) and $920^{\circ} \mathrm{F}$ (upper curve). 


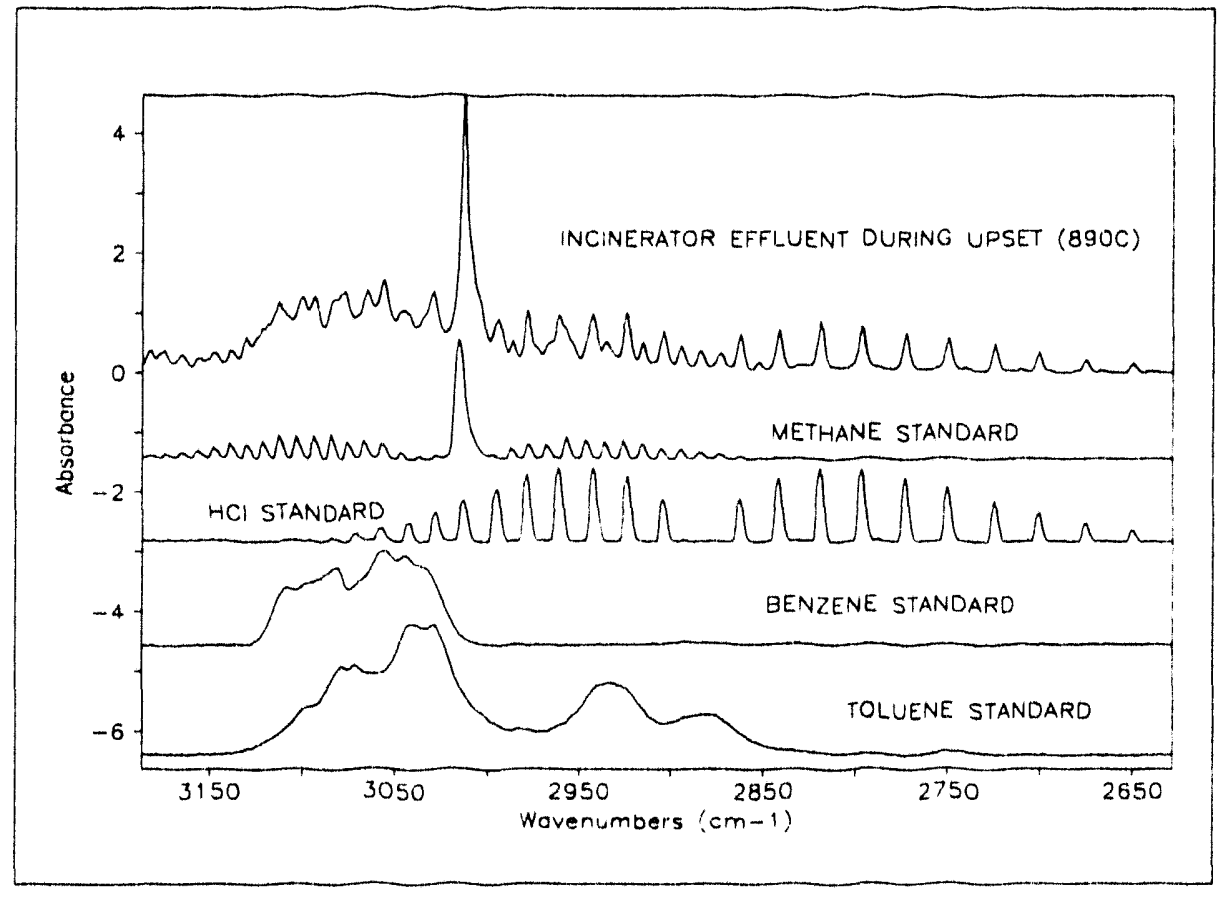

Figure 3. Comparison of a Spectrum Obtained During Incinerator Upset and Selected Analytical Standards.

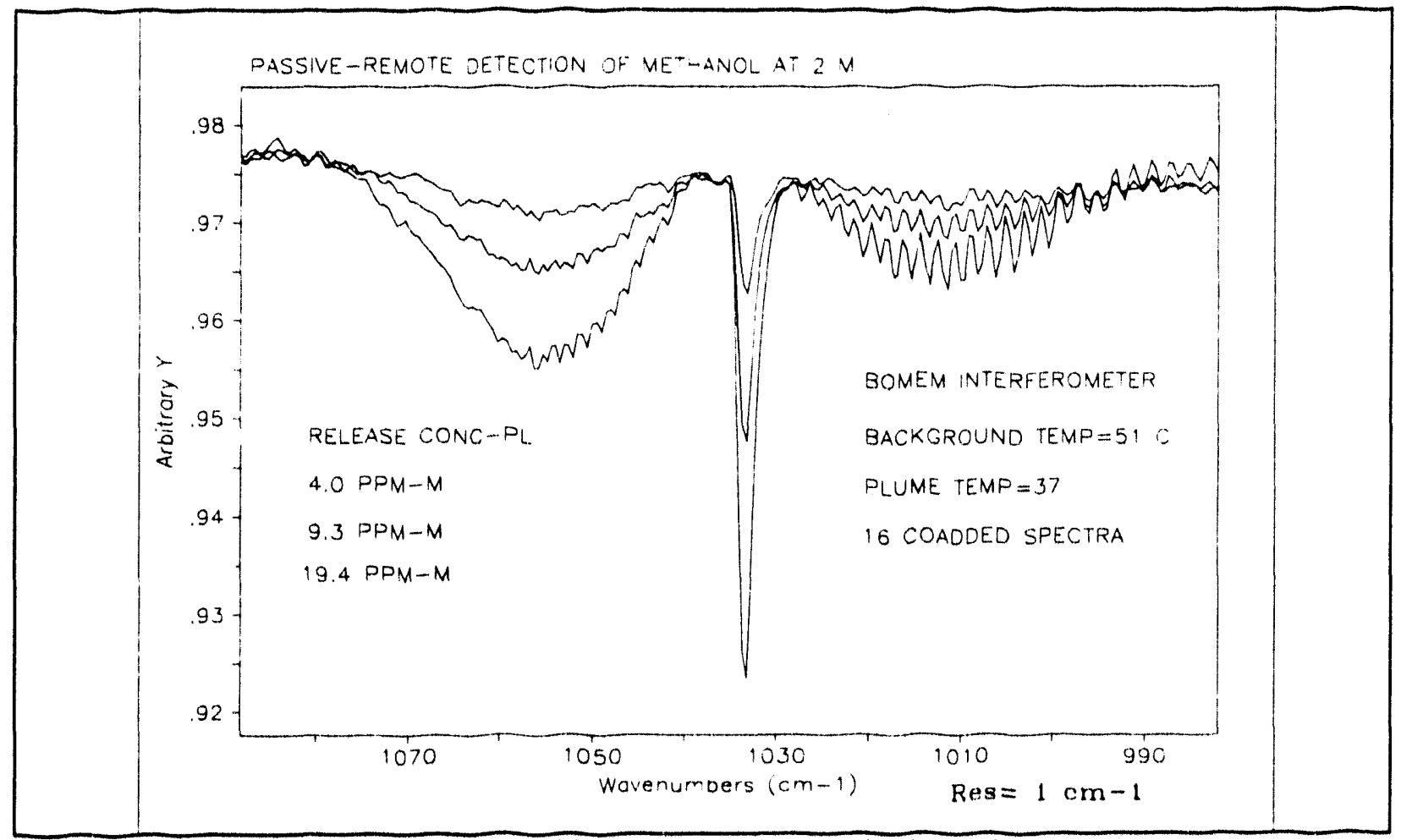

Figure 4. Passive-remote Detection of Methanol Releases in the Laboratory. 


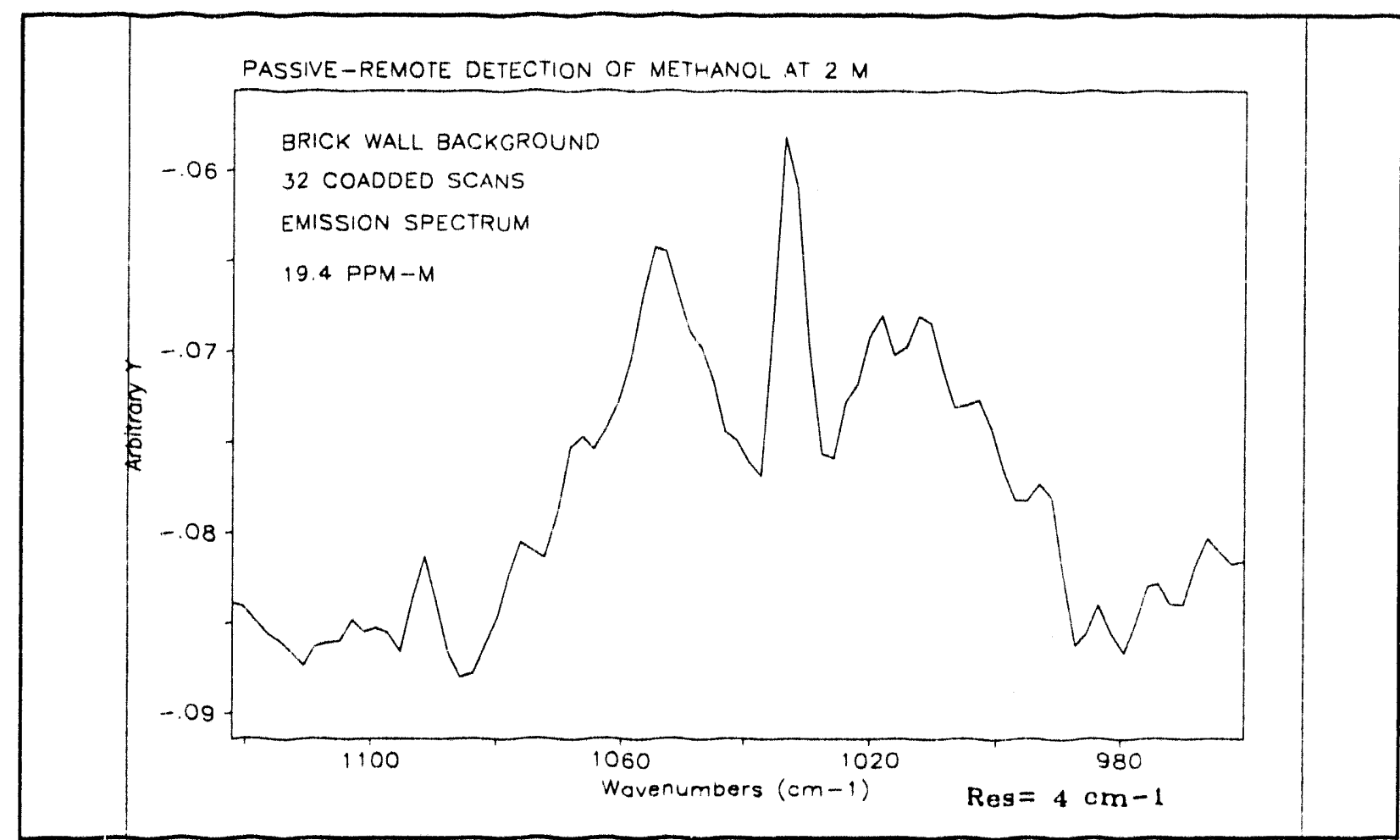

Figure 5. Emission Spectrum of a $10 \mathrm{~cm}$ Methanol released in front of a Brick Wall detected from $2 \mathrm{~m}$.

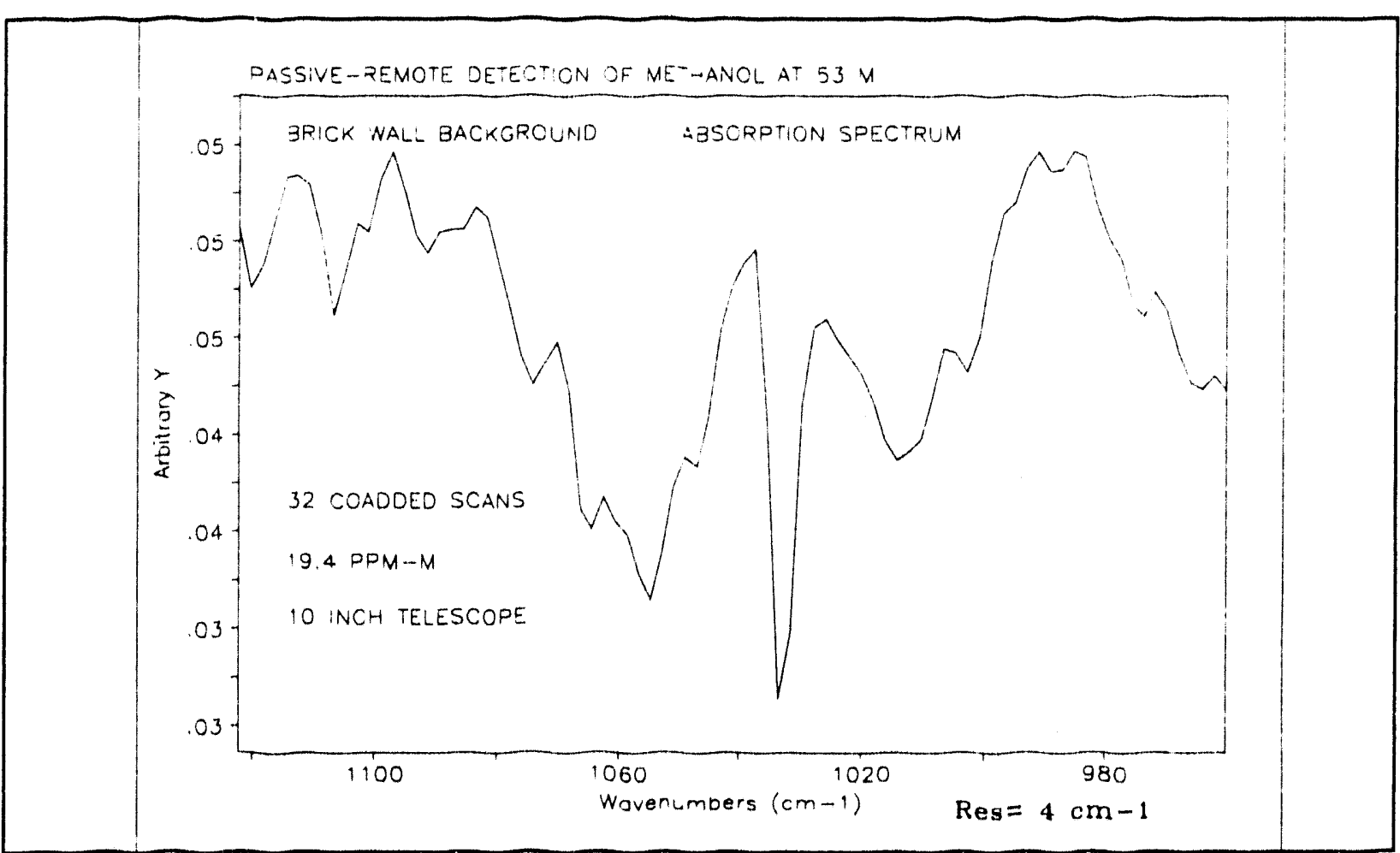

Figure 6. Absorption Spectrum of a $10 \mathrm{~cm}$ Methanol Release in front of a brick Wall Detected from $53 \mathrm{~m}$. 


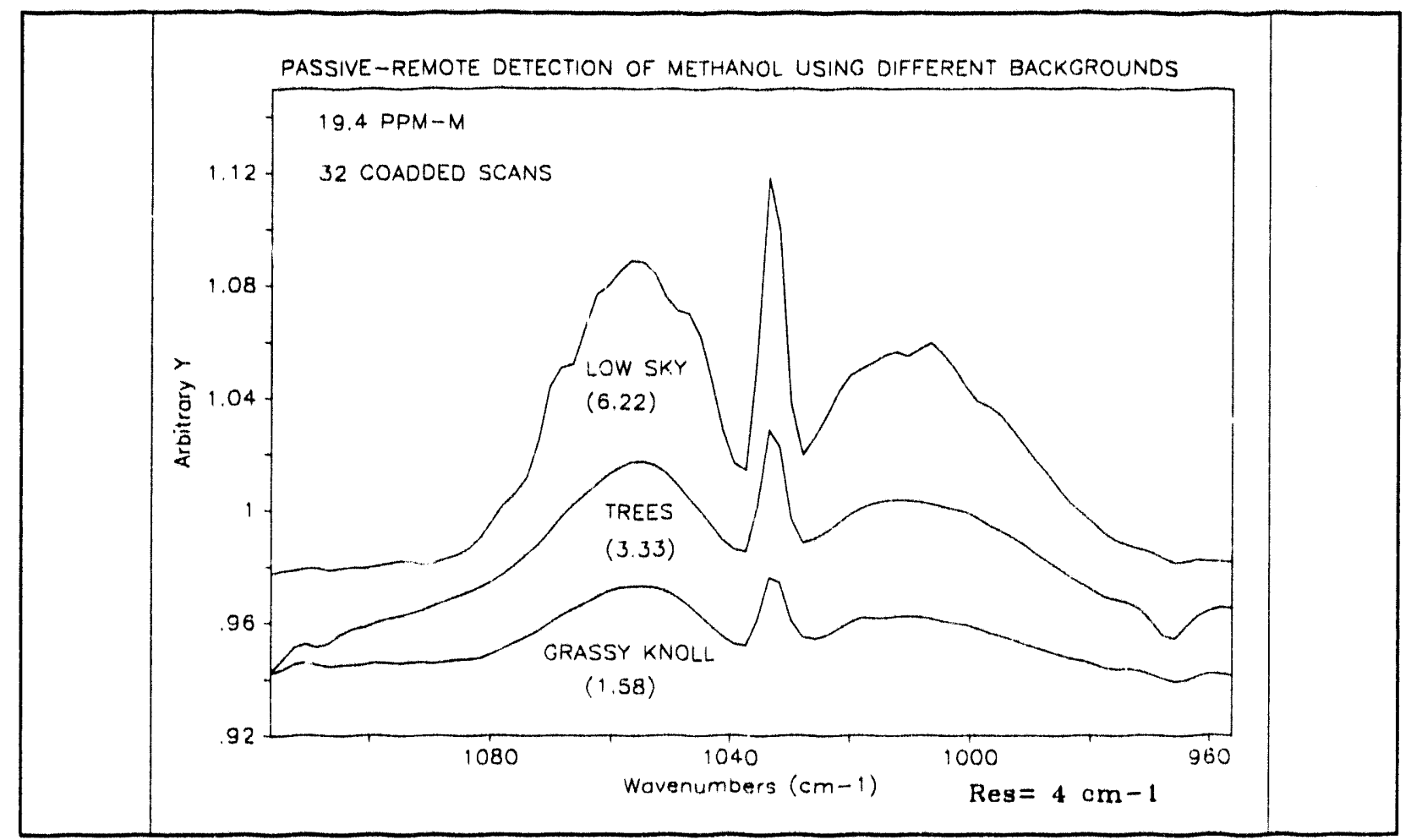

Figure 7. Passive-Remote Detection of Methanol Under Different Background Conditions.

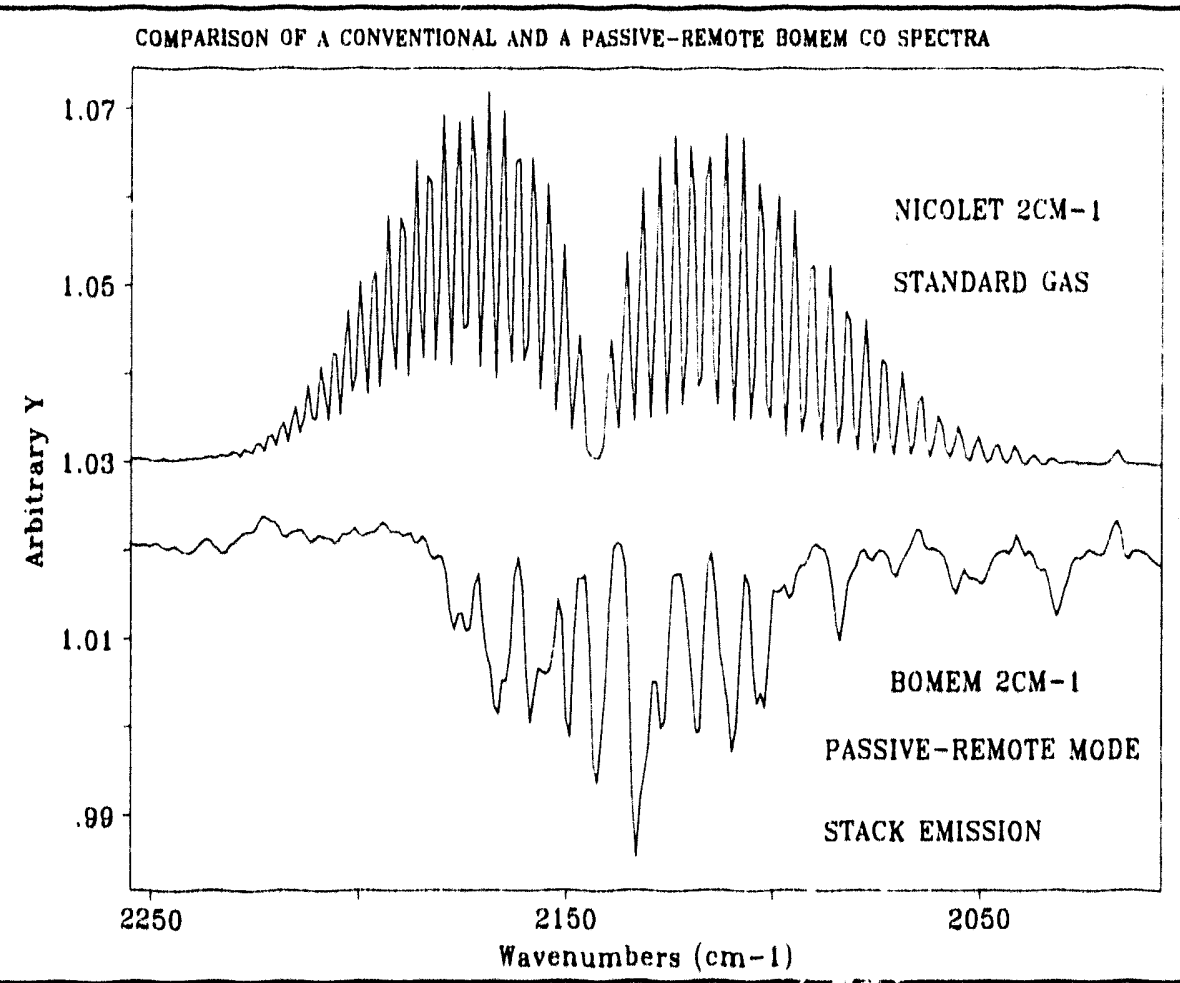

Figure 8. Infrared Spectra of CO Obtained from a Power Plant and in the Laboratory. 


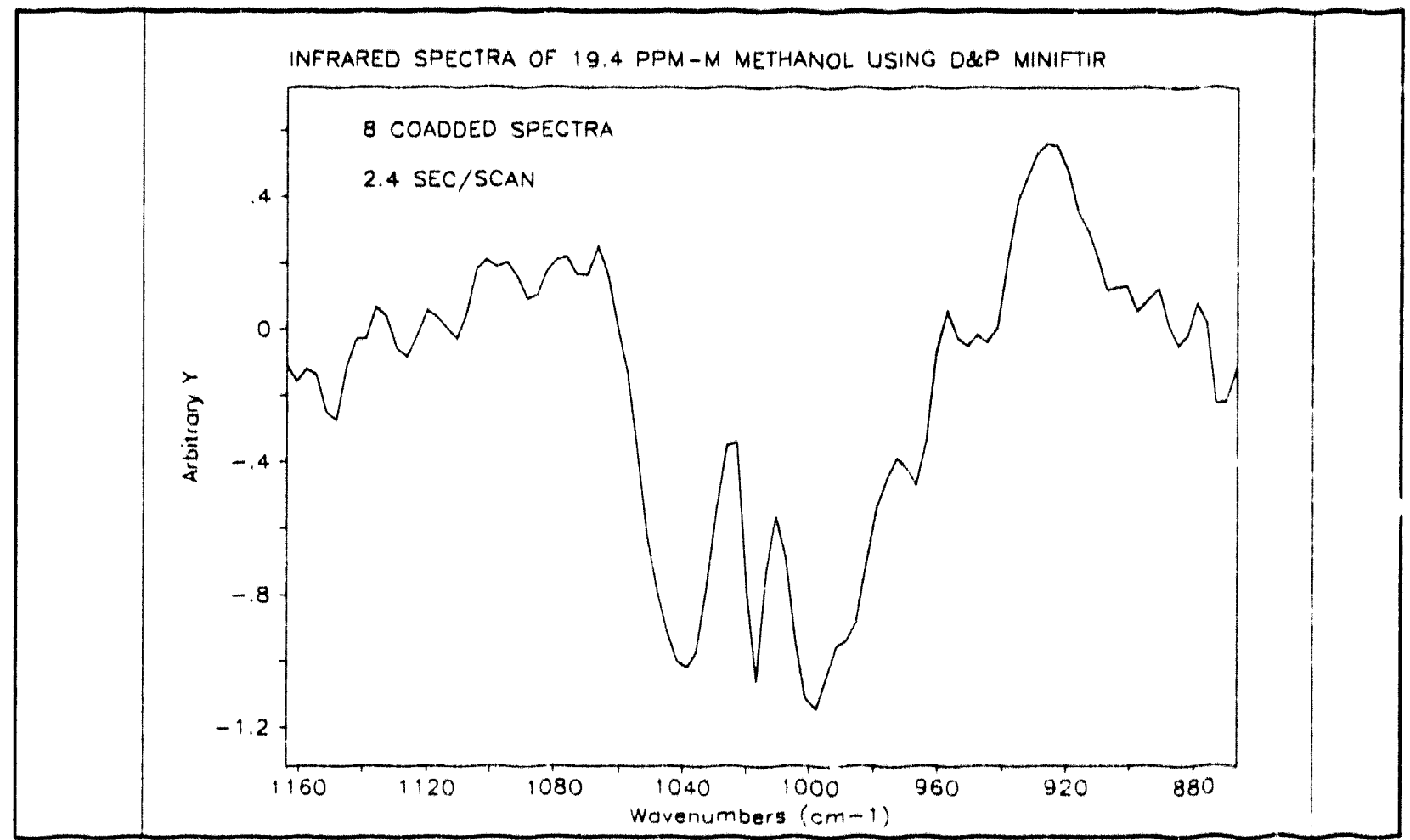

Figure 9. Infrared Spectrum of a 19.4 Methanol Release Obtained Using a DP MiniFTIR Spectrometer. 

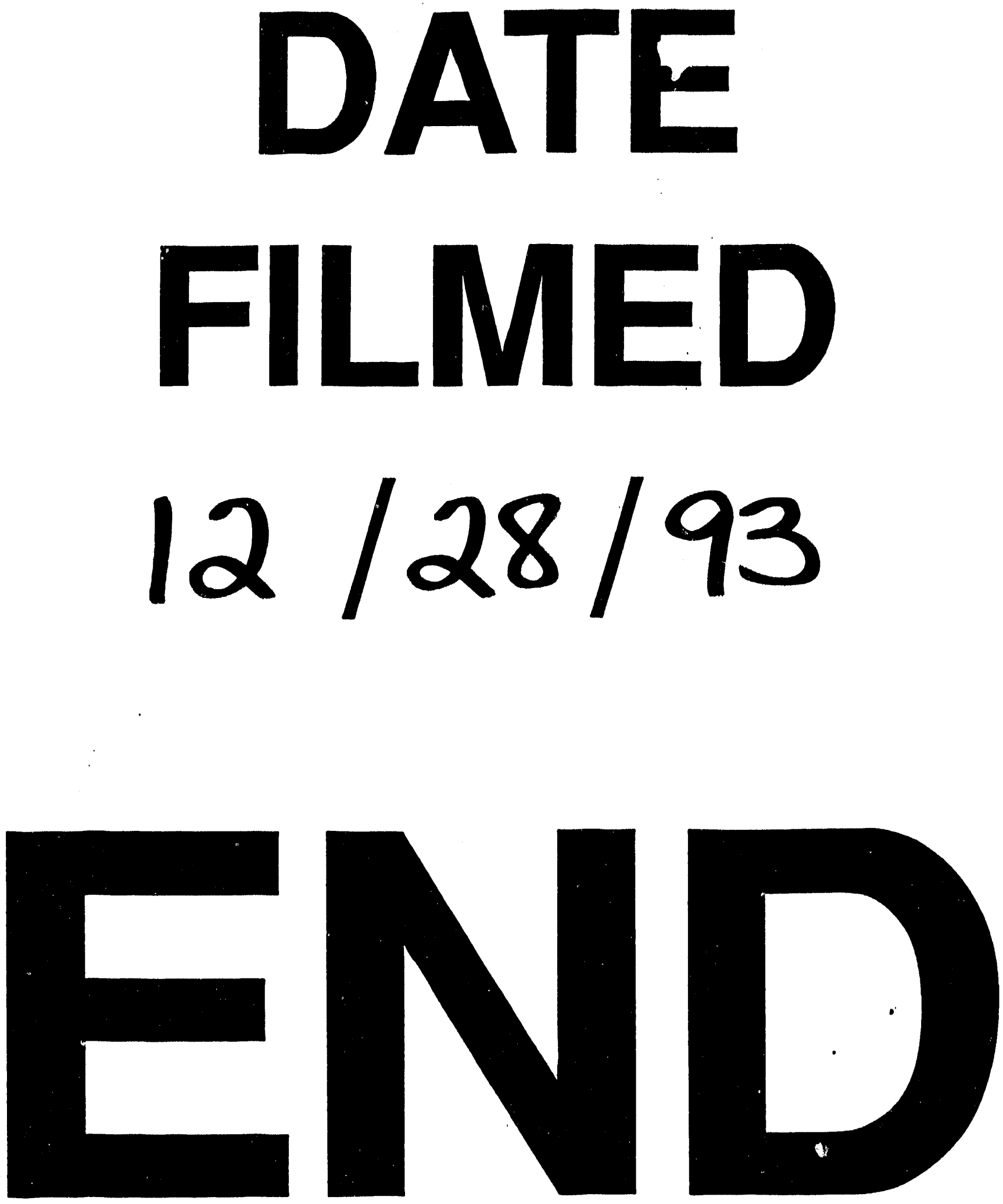
Hen

A

.

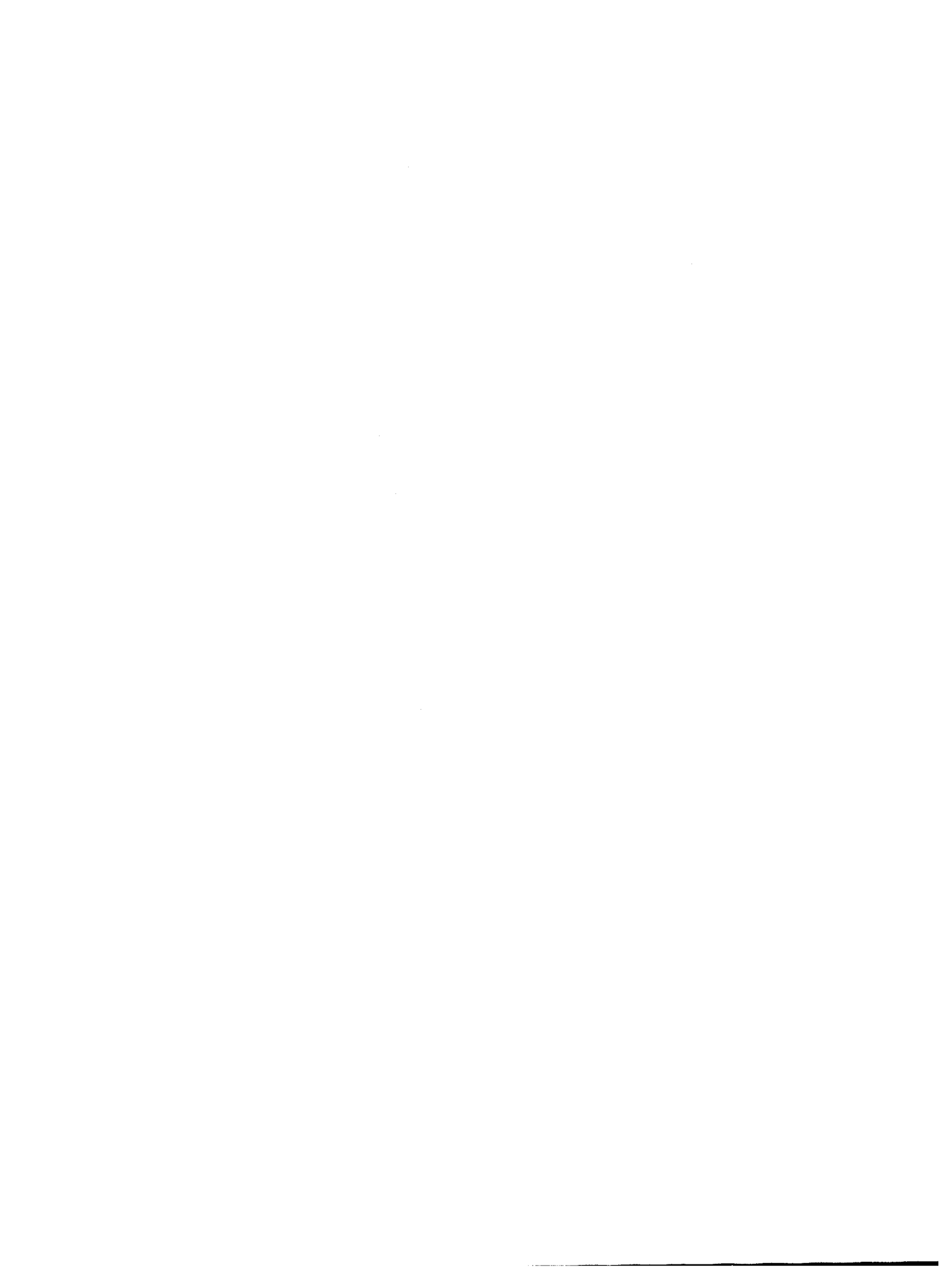
$-$ 